\title{
Trigger of a migraine headache among Thai adolescents smartphone users: a time series study
}

\author{
Wanna Chongchitpaisan ${ }^{1, *}$ (D), Phongtape Wiwatanadate ${ }^{2, *}$, Surat Tanprawate ${ }^{2}$, Assawin Narkpongphan ${ }^{2}$, Nipapon \\ Siripon ${ }^{3}$ \\ ${ }^{1}$ Institute of Occupational and Environmental Medicine, Department of Medical service, Ministry of Public Health, Thailand \\ ${ }^{2}$ Faculty of Medicine, Chiang Mai University, Chiang Mai, Thailand \\ ${ }^{3}$ Faculty of Engineering, Chiang Mai University, Chiang Mai, Thailand \\ *Correspondence: pwiwatanadate@gmail.com
}

Received: May 30, 2020 Accepted: January 31, 2021

\begin{abstract}
The study aims to investigate the effect of modern technology in the trigger of migraine headaches in high school students by using generalized estimating equation analysis. The prospective time series study was conducted in one hundred and forty-five smartphones using high school students in Chiang Mai Province who each completed a headache diary giving a total of 12,969 data entries. The smartphone output power was measured and recorded by a smartphone application. Smartphone use, sleep quality, anxiety, and depression also were assessed. Results revealed that the prevalence of repeated headache be $13.4 \%$ and migraine $16.9 \%$. The migraine had the strongest association with handfree device use and internet use, followed by age and anxiety. Furthermore, the strongest effect of smartphone output power in triggering a migraine was found at $\leq 1.79 \times 10^{-5}$ and $1.80-1.99 \times 10^{-5} \mathrm{~mW}$ ranges. Meanwhile, Lag_6 of daily smartphone output power exposure produced the migraine effect in a reverse dose-response manner. The smartphone electromagnetic radiation was a primary migraine trigger. The study results led to the recommendation to avoid triggers by use of smartphone talking with hand-free devices to prevent a recurrent migraine.
\end{abstract}

Keywords: trigger, migraine, adolescents, smartphone users

\section{Introduction}

Migraine is one of the most frequent and severe headaches caused by stimulation deep in the brain causing inflammation and pain around nerves and blood vessels in the brain and classified in one group of primary headache by international classification of headache disorders (ICHD) [1]. Previous studies reported light, odor, hunger, weather and smoke as more common triggers of migraine [2]. Migraine is triggered by the stressors which have response mechanism to repeat the stressor of the same kind [3] and increase response to other mediators, manifested as central sensitization. Adolescent most of the time is exposed to various triggers and excessive use of electronic devices that found a risk for ordinary headaches and migraine [4]. Migraine and particularly repeated or recurrent migraine might impose a recognizable burden on the suffers in their adolescence in terms of their impaired health, quality of life and academic performance as well as cause their parent's financial burden in terms of treatment costs [5,6]. A previous study supported comprehensive migraine treatment programs with avoidance of trigger factors through appropriate lifestyle change to reduce headache frequency [2].

The smartphone is manufactured with modernized technology and is popularly used to meet people's various needs. In Thailand, the overall values of the communications market in 2011 grew by $9.2 \%$ and the smartphone market was forecasted to increase by $24.9 \%$ [7]. Previous studies on the health effects of smartphone use generally reported findings related to headaches (63.2\%) [8]. The smartphone output power is an important indicator in assessing electromagnetic energy outside the body related to exposure levels and the amount of energy absorbed in tissues [9]. The smartphone output power was used in real time by software in the smartphone and measuring output power out of smartphone by software or computer program but not measuring individual levels because measurements are taken with the device [10,11].

A smartphone is an electromagnetic emission source closest to the head and the human nerves which are the electrical parts of human body and far from being in a state of equilibrium. Although the emitted electromagnetic energy is below the maximum standard value, it could more or less affect human. Electromagnetic radiation was found to affect the areas of thalamus, raphe magnus nucleus, and spinal cord by reducing the neurotransmitters such as acetylcholine, neuronal 
nAChR, and those that have an important role in inhibiting pain [12]. This radiation was also found to affect the functioning of the dopamine-opiate system resulting in lowering the number of cells of the neurotransmitter, to the extent that it cannot perform the function of a pain suppresser [12]. All of these are the main mechanisms of headaches. Smartphone electromagnetic energy might act as a repeated stressor which triggers migraine.

The effects of electromagnetic radiation in producing migraine remain unclear. The studies of smartphone use and its migraine impact will provide data for recommending safe smartphone use which perhaps can help decrease migraine headache frequency.

\section{Materials and Methods}

The sample size was calculated based on $10 \%$ prevalence of headache by mobile phone used [14]. The sample of 966 high school students in Chiang Mai, Thailand completed questionnaires in the first phase. This study selected 200 samples from 966 high school students in the first phase based on the inclusion criteria such as the subjects owned at least one smartphone and no family history of migraines, no daily health-related behaviors including liquor, coffee or tea consumption, and smoking, had no diseases or health problems diagnosed by the doctors, nor were they undergoing any the treatment. The exclusion criteria were that they had hospital anxiety and depression (HAD) scores equal to 11 points and more in the first phase and no severe illnesses or injuries. The prospective time series study was conducted during from October to December 2015. It was approved by the Ethics Committee for Human Research, Faculty of Medicine at Chiang Mai University. The 200 samples were asked to record headache and sleep quality by a smartphone application daily (Figure 1). Finally, 12,696 records were obtained from totally 145 students. The 55 samples withdrew from the study because they had been loaded with their classes and some failed to send information. The headache was assessed by daily headache questionnaire that was conducted based on ICHD version 3 beta criteria and scored to determine headache type according to the diagnosis algorithm [13]. The first headache type is classified as migraines characteristics and groups are divided by scores where migraines $=3$, potential migraines $=2$ and scores of less than uncategorized patients $=2$. For the subjects with scores less than or equal to 2 , their scores were recalculated by tension type headache (TTH) characteristics. The recalculated scores were then used for category assignment of the subjects as in TTH group if score $=3$, in potential migraine group if score $=2$, and in undetermined headache group if score is lower than 2 . This means those subjects with original score of exactly 2 will still be in the potential migraine group if their recalculated scores failed to meet TTH criteria. The sleep quality was assessed by the Pittsburg sleep quality index (PSIQ) which has been translated into Thai. The HAD form has been used to assess depression and anxiety and the use smartphones was also assessed by diary questionnaires. The total data was regularly recorded every day over a period of two to four months (60-120 days) by the smartphone application. Smartphones measured output power from smartphone antenna to the nearest area and the application has requested access to the smartphone output power via the program's framework by setting to save every five minutes and sent to the researcher by email. The smartphone output power was continuous data with non-normal distribution and divided into three groups.

Sampling High school student include 3 grades to complete the questionnaire, 1,058

\section{$\downarrow$}

Samples returned and completed the questionnaire, 966

$\boldsymbol{7}$

Samples in $1^{\text {st }}$ phase were selected to $2^{\text {nd }}$ phase by inclusion and exclusion criteria, 200

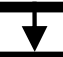

145 samples completed daily record over a period of 60-120 days, 12,969

Figure 1 The sampling procedure and sample response flow chart 


\section{Statistical Analysis}

The data resulting in 12,969 records which then were coded and analyzed using Statistical Package for Social Science (SPSS) software version 20 to obtain frequency, arithmetic mean, and standard deviation. Relationships between smartphone output power and migraine headaches were investigated with odds ratio (OR) and their 95\% confidence intervals (95\% confidence intervals [CIs]) with $p$-value $<0.05$ considered statistically significant. Data analysis was conducted using generalized estimating equations (GEE) which can handle the confounding effects of such factors as demographic data, sleep quality, anxiety, depression and smartphone use, and no interaction effect was found. GEE analysis is suitable for dependent variables with correlated responses because data were collected from the same person. In the analysis, therefore, the correlational structure was set for dependent variables by considering the quasi-likelihood under an independence model criterion (QIC) where low QIC scores indicated that the correlational structure was good and corrected QIC (QICC) was used to compare the models under the same correlational structure. Low QICC scores indicated that the model was a fit.

\section{Results}

The study found that the majority of the samples were female, 17.4 years old on average with a normal health condition. The prevalence of person-day headaches to be $13.4 \%$. In most cases, the headaches were TTH, $74.1 \%$, followed by unidentified and migraine types at $16.9 \%$ and $9.0 \%$, respectively (Table 1 ).

The data on smartphone output power was adjusted by the value of error measured from each device brand to normalize the value for all device brands (Table 2). The smartphone output power (SOP) values observations were then divided into three ranged groups: $\leq 1.79 \times 10^{-5}, 1.8-1.99 \times 10^{-5}$, and $\geq 2.0 \times 10^{-5} \mathrm{~mW}$ (Table 3 ). The most common range of SOP to which the samples were exposed was found to be $\geq 2.0 \times 10^{-5} \mathrm{~mW}, 83.2 \%$ of the observations. SOP in the $1.8-1.99 \times 10^{-5} \mathrm{~mW}$ range appeared least prevalent, only $1.5 \%$ and SOP in the $\leq 1.79 \times 10^{-5} \mathrm{~mW}$ range prevailed in $15.3 \%$ of the observations.

Table 1 Characteristics of person-day headache of participants presented as percent.

\begin{tabular}{lc}
\hline \multicolumn{1}{c}{ Variable } & N (\%) \\
\hline Headache (time) & \\
Yes & $1705(13.4)$ \\
No & $10991(86.6)$ \\
Headache type & \\
Migraine & $153(9.0)$ \\
Tension type headache (TTH) & $1264(74.1)$ \\
Undetermined & $288(16.9)$ \\
\hline
\end{tabular}

Table 2 The error output power of application by spectrum analyzer

\begin{tabular}{|c|c|c|c|c|c|c|c|c|c|c|}
\hline \multirow{3}{*}{ Brand device } & \multicolumn{9}{|c|}{ Mode } & \multirow{3}{*}{$\begin{array}{l}\text { Average } \\
\text { error } \\
\text { (dB) }\end{array}$} \\
\hline & \multicolumn{3}{|c|}{ Standby (dB) } & \multicolumn{3}{|c|}{ Call (dB) } & \multicolumn{3}{|c|}{ Talk (dB) } & \\
\hline & App & Spec & Error & App & Spec & Error & App & Spec & Error & \\
\hline Brand 1 & -61.00 & -35.65 & -25.35 & -58.00 & -35.65 & -22.35 & -56.00 & -33.65 & -22.35 & -23.35 \\
\hline Brand 2 & -65.50 & -54.10 & -11.40 & -59.00 & -45.50 & -13.50 & -53.50 & -43.50 & -13.00 & -12.60 \\
\hline Brand 3 & -57.00 & -37.65 & -19.35 & -53.00 & -36.65 & -16.35 & -51.00 & -34.65 & -16.35 & -17.35 \\
\hline Brand 4 & -63.00 & -37.65 & -25.35 & -55.00 & -35.65 & -19.35 & -51.00 & -34.65 & -16.35 & -20.35 \\
\hline Brand 5 & -72.50 & -62.50 & -10.50 & -59.50 & -49.50 & -10.50 & -57.60 & -47.50 & -10.40 & -10.50 \\
\hline
\end{tabular}

App $=$ Measured by application, Spec $=$ Measured by spectrum analyzer (gold standard)

Table 3 Number of smartphone output power (SOP) grouped by time cycles and daily dose.

\begin{tabular}{cccccc}
\hline $\begin{array}{c}\text { Output power } \\
\left(\times \mathbf{1 0} \mathbf{0}^{-5} \mathbf{~} \mathbf{W}\right)\end{array}$ & Daily dose (\%) & Morning (\%) & Daytime(\%) & Evening (\%) & Nocturnal(\%) \\
\hline$\leq 1.79$ & $1943(15.3)$ & $3597(31.4)$ & $2479(20.1)$ & $2303(18.8)$ & $2648(20.9)$ \\
$1.8-1.99$ & $186(1.5)$ & $226(2.0)$ & $120(1.0)$ & $79(0.6)$ & $301(2.4)$ \\
$\geq 2.0$ & $10567(83.2)$ & $7646(66.7)$ & $9710(78.9)$ & $9896(80.6)$ & $9747(76.8)$ \\
\hline
\end{tabular}


To control the confounding effects, we conducted a statistical test to evaluate the relationship between various factors and found no interaction effect among them. Additional computation was made to adjust the effects of such potential confounders as demographic characteristics, and smartphone use. Auto regression 1 (AR1) was set as the correlational structure due to its lowest QIC (Tables 4 and 5). Apparently, the SOP in $\leq 1.79 \times 10^{-5}$ and $1.80-1.99 \times 10^{-5} \mathrm{~mW}$ ranges had the strongest correlation with migraines (ORadj $=2.02 ; 95 \%$ CI: $1.17-3.49$ and ORadj $=3.25 ; 95 \%$ CI: $1.65-6.42)$ (Table 4$)$ and were riskier than that in the $\geq 2.00 \times 10^{-5} \mathrm{~mW}$ range. The undetermined category of headaches was also correlated with the SOP $1.80-1.99 \times 10^{-5} \mathrm{~mW}$ range (ORadj $\left.=2.32 ; 95 \% \mathrm{CI}: 1.23-4.34\right)$. Meanwhile, TTH did not occur as a response to SOP at any range of exposure. The other factors, hand-free use and internet use had the strongest association with TTH (ORadj $=3.72 ; 95 \% \mathrm{CI}$ : 2.49-5.56 and $\mathrm{OR}_{\mathrm{adj}}=2.15 ; 95 \%$ CI: 1.75-2.64), migraine (ORadj $=3.96 ; 95 \%$ CI: 1.21-12.87 and ORadj $=2.06$; 95\% CI: 1.20-3.51) and undetermined headache (ORadj $=1.92 ; 95 \%$ CI: 1.11-3.29 and ORadj $=2.33 ; 95 \%$ CI: 1.71-3.19). Furthermore, the results indicated that the factors of younger age and anxiety were related with migraines (ORadj $=1.79 ; 95 \%$ CI: 1.38-2.31 and ORadj $=1.12 ; 95 \%$ CI: $1.06-1.19$ )

\section{Discussion}

The present study with daily data shows current person-day headaches to be $13.4 \%$, and migraine type to be $9.0 \%$. The results were consistent with the findings from the first phase of the study, involving the entire student population. The prevalence of headache and migraine type was $47 \%$ and $20.4 \%$ in Brazil [15], which is, however, different from studies conducted elsewhere. A study in Sweden using a daily headache record revealed migraines were 24.9\% [16]. The 2002-2012 survey in USA revealed the prevalence of migraine headache to be 12.2\% [17]. Headache prevalence varies across geographic areas, cultures, and the sample groups, as well as the criteria for classifying types of headache [15].

Table 4 Odds ratio (OR) of migraine type and its 95\% confidence intervals (CI) for each factor and daily dose adjusted for all other factors using generalized estimating equations (GEE) (Auto regression 1; Quasi likelihood under an independence model criterion $[\mathrm{QIC}]=1314.86$; corrected $\mathrm{QIC}[\mathrm{QICC}]=1309.60$ )

\begin{tabular}{|c|c|c|c|c|c|c|c|}
\hline \multirow{2}{*}{ Factor } & \multicolumn{2}{|c|}{ Migraine headache } & \multirow{2}{*}{$\begin{array}{l}\text { Crude } \\
\text { OR }\end{array}$} & \multirow{2}{*}{$\begin{array}{l}\text { Adjusted } \\
\text { OR }\end{array}$} & \multicolumn{2}{|c|}{$95 \% \mathrm{CI}$} & \multirow{2}{*}{$\begin{array}{c}\mathrm{p}- \\
\text { value }\end{array}$} \\
\hline & Yes & No & & & Lower & Upper & \\
\hline Age $($ mean \pm SD) & $16.9 \pm 0.9$ & $17.4 \pm 1.0$ & 1.80 & 1.79 & 1.38 & 2.31 & $<0.01$ \\
\hline Anxiety score (mean \pm SD) & $3.0 \pm 3.0$ & $1.8 \pm 2.4$ & 1.12 & 1.12 & 1.06 & 1.19 & $<0.01$ \\
\hline $\begin{array}{l}\text { Lag_6 dose }\left(\times 10^{-3} \mathrm{~mW}\right) \\
(\text { mean } \pm \mathrm{SD})\end{array}$ & $0.72 \pm 1.4$ & $2.1 \pm 16.3$ & $6.3 \times 10^{-44}$ & $1.11 \times 10^{-38}$ & $4.55 \times 10^{-69}$ & $2.69 \times 10^{-8}$ & 0.01 \\
\hline Total & 153 & 12537 & & & & & \\
\hline Internet use (Yes/ No) & $1416 / 11280$ & $2.0 / 1.1$ & 1.89 & 2.06 & 1.20 & 3.51 & $<0.01$ \\
\hline $\begin{array}{l}\text { Hand-free use (No/ } \\
\text { Frequent) }\end{array}$ & $10477 / 951$ & $1.3 / 0.5$ & 3.26 & 3.96 & 1.21 & 12.87 & 0.02 \\
\hline \multicolumn{8}{|l|}{ Dose group $\left(\times 10^{-5} \mathrm{~mW}\right)$} \\
\hline$\leq 1.79 / \geq 2.00$ & $1943 / 10567$ & $2.1 / 1.0$ & 2.00 & 2.02 & 1.17 & 3.49 & 0.01 \\
\hline $1.80-1.99 / \geq 2.00$ & $186 / 10567$ & $2.7 / 1.0$ & 4.08 & 3.25 & 1.65 & 6.42 & $<0.01$ \\
\hline
\end{tabular}

Adjusted by age, body-mass index (BMI), vision, anxiety, depression, Pittsburg sleep quality index (PISQ), internet use, hand free use, brand device, and smartphone output power (SOP).

Table 5 Odds ratio (OR) of headache type and their 95\% confidence intervals (CIs) for each factor and lag dose adjusted for all other factors using generalized estimating equations (GEE)

\begin{tabular}{|c|c|c|c|c|c|c|c|}
\hline \multirow{2}{*}{ Parameter } & \multirow{2}{*}{$\operatorname{Exp}(B)$} & \multicolumn{2}{|c|}{ 95\% CI for Exp (B) } & \multirow{2}{*}{ p-value } & \multirow{2}{*}{$\begin{array}{c}\text { Correlation } \\
\text { structure }\end{array}$} & \multirow{2}{*}{ QIC } & \multirow{2}{*}{ QICC } \\
\hline & & Lower & Upper & & & & \\
\hline \multicolumn{8}{|c|}{ Migraine type } \\
\hline Lag_6 & $1.106 \mathrm{E}-38$ & $4.548 \mathrm{E}-69$ & $2.690 \mathrm{E}-08$ & 0.01 & AR1 & 1346.608 & 1341.171 \\
\hline Lag_6 & $3.988 \mathrm{E}-46$ & $8.918 \mathrm{E}-83$ & $1.784 \mathrm{E}-09$ & 0.02 & Exchangeable & 1347.436 & 1341.779 \\
\hline
\end{tabular}

Adjusted by age, body-mass index (BMI), vision, anxiety, depression, Pittsburg sleep quality index (PISQ), internet use, hand free use, and brand device. QIC = Quasi likelihood under an Independence Model Criterion; QICC = Corrected QIC; AR1 = auto regression 1 
The present study showed that a year younger student compared to a year older one was likely to face a relatively greater degree of migraine and undetermined headache. This result is in contrast with most previous studies which found headache prevalence to vary positively with age [18], particularly the migraine type headache. The results from the present study are in line with those found from the first phase investigation on factors associated with headache from mobile phone use that young age had implication for mobile phone associated headache. A study in Korea surveys revealed as high as $31 \%$ of children aged 8-10 owned and used mobile phones and found the average age of children first using a mobile phone decreased from 12.5 years old in 2008 to 8.4 years old in 2011 implying a tendency for children to own and use mobile phones at younger ages [19].

High scores of PSQI are found to be linked with migraines [20,21] and sleep quality has been found to be a highly explanatory factor for frequent headache attacks [21], particularly migraine, triggered by the hypothalamus which is linked with limbic system, retino hypothalamic tract, and brain-stem aminergic nuclei [22], as well as with periaqueductal gray (PAG) matter. Rapid-eye movement or sleep-off is activated by the orexin. Orexin works by stimulating neurons in the ventrolateral part of the peri-aqueductal gray matter, which normally functions as an inhibitor of the antinociceptive activity in the trigeminal nucleus caudalis, thereby resulting in causing a migraine attack [23]. Evidences exist that migraines can be affected by neurotransmitters, serotonin, dopamine, and melatonin and the anti-inflammatory system and migraine will affect the level of melatonin [20]. The result from the present study confirms the association between sleep quality and migraine.

Internet use is a risk factor of migraine, TTH and undetermined headache (ORadj $=1.98-2.41 ; 95 \%$ CI: $1.20-3.51$, $\mathrm{OR}_{\mathrm{adj}}=2.15 ; 95 \% \mathrm{CI}: 1.75-2.64$ and $\mathrm{OR}_{\mathrm{adj}}=2.33 ; 95 \% \mathrm{CI}$ : 1.71-3.19). Talking on smartphones in both internet and cellular modes often involves holding the device close to the head, and the electromagnetic radiation from smartphone to which the users are exposed induces changes in biological reaction, change of protein in the brain, and causes nervous system problems, especially headache symptoms [24]. There is also a finding from the present study that not using hand-free devices while talking on smartphone produces the strongest effect in causing migraine and TTH (ORadj $=3.96 ; 95 \%$ CI:1.21-12.87 and ORad) $=3.72 ; 95 \%$ CI: 2.49-5.56).

Smartphone use among sampled students was considered on the basis of SOP which was measured and stored in the device and was viewed with the use of an application. The SOP values received in the $1.8-1.99 \times 10^{-5} \mathrm{~mW}$ range appeared to be the least prevalent, only $2.4 \%$ of the observations, taking place mostly during nighttime. Previous studies indicated most teenagers, $62-72 \%$, used advanced smartphones in the evening and at night, after 9 pm and during 0-3 a.m., with 34$55 \%$ of the use for texting, social media, and $24 \%$ for playing games [25]. SOP values in this study were thus lower than the values of smartphone electromagnetic radiation in other studies which used an external metering device and might be affected upward by the radiation from other sources. In this study, the maximum SOP was $1.55 \mathrm{~mW}$ which was different from the study of Lonn et al. which showed the most frequently used power levels were the highest at $33 \mathrm{dBm}$ and the lowest at $5 \mathrm{dBm}$ [26], and the mean TDMA and GSM power output values were 18.2 and $14.1 \mathrm{dBm}$. In the study of Kelsh et al. and Gosselin the average output power was between $0.001-0.63 \mathrm{~mW}[10,27]$. This value concurred with the average power consumption of a human cell, at $1 \times 10^{-9} \mathrm{~mW}$ [28].

The present findings revealed that migraine headaches were correlated with smartphone output power at the ranges of $\leq 1.79 \times 10-5$ and $1.80-1.99 \times 10^{-5} \mathrm{~mW}$. The correlation was found to be non-linear, which agreed with the previous experimental studies on exposure to microwave frequency radiation which found the response to take place only at specific values or in a specific range called window effect. Similarly, an experimental study of Frey et al. and Merritt et al. found that the blood brain barrier responds to microwave frequency radiation only at $2.4 \mathrm{~mW} / \mathrm{cm}^{2}$ and $0.2 \mathrm{~mW} / \mathrm{cm}^{2}$ intensity [29]. The mechanism of headache response to smartphone electromagnetic radiation is often driven by the dysfunction of the endogenous pain control system affecting the areas of thalamus, raphe magnus nucleus and spinal cord by decreasing neurotransmitters such as acetylcholine, neuronal nicotinic acetylcholine receptors playing an important role in inhibiting pain [29].

Furthermore, our study found migraine to respond to the delayed effect of daily SOP after a seven-day exposure (lag_6) in a reverse dose-response manner. The repeated exposure to electromagnetic radiation for a long period results in the accumulation of response and the long-time response, which is consistent with the findings of Merritt et al., in the year 1977 [29]. The 1977 study was conducted in experimental rats with exposure to electromagnetic radiation, seven hours a day for 30 days, and found the epinephrine response in the brain to increase on the twentieth day after the exposure and returned to normal on the thirtieth day. The results of high intensity revealed an increase in norepinephrine, dopamine, and serotonin on the fifth day after exposure which, however, reduced after the continued or repeated exposure. However, there is no clear explanation on the mechanism for the occurrence of delayed response to electromagnetic exposure which might be due to the adaptive process of the nervous system to return to equilibrium or due to allostatic load [30]. Yet, the repeated exposure is synonymous with the continued accumulation and thus causes the nervous system to respond to an accumulated effect.

Characteristically, a migraine occurs as the result of the brain's nervous system using an adaptive process as a response mechanism to cope with repeated stress. The adaptive process involves the continuative and cumulative alteration 
in cells and system which leads to either the restoration of equilibrium (allostasis) or allostatic load, if the adaptive process takes place abnormally [30]. The migraine maladaptive process results in the system's failure to habituate to repeat the stressor of the same kind [3] and this activates increased response to other mediators, manifested as central sensitization leading to lower resistance to pain, and sensitivity in response to the same stressor [31]. Certain responses reflect a protective adaptive process, for example: allodynia, phonophobia, photophobia, osmophobia, and allodynia, which are pain triggered by the stressors at a normal level [32,33]. Thus, the findings from the first phase of this study, that headache associated with mobile phone use is not severe [14], can be used to explain that migraine has a response to low output level and delayed effect in the form of reverse dose-response, and that it is also an output effect.

Finally, the findings from the present study point out that smartphone electromagnetic radiation is likely to be the trigger of migraine type headache. To be considered as a trigger [22], the following conditions must occur: 1) a trigger must be able to reach blood vessels or many receptors in the nervous system, to cause the biochemical reactions; 2) a trigger must have a reasonable linkage with the receptors. Previous studies revealed that mechanism of headache response to microwave electromagnetic radiation was inducing the response from various neurotransmitters and endogenous-opioids [24,29]. 3) There is a definite amount of exposure to make the response observable. Study findings revealed that migraine responds to smartphone output power in the window effect pattern $\left(1.80-1.99 \times 10^{-5} \mathrm{~mW}\right.$ and $\left.\left.\leq 1.79 \times 10^{-5} \mathrm{~mW}\right) ; 4\right)$ There might be co-factors for triggering effect like the use of smartphone for a long-time conversation; 5) Triggers on the central nervous system effects must be able to penetrate or damage the blood-brain barrier. The existence of empirical evidence has proven that microwave electromagnetic radiation causes alterations in the blood-brain barrier [29]. Thus, smartphone electromagnetic radiation is a new trigger of migraine.

The headaches in this study as reported by students and classified type of headache by a diagnostic algorithm might be a limitation of this study. Furthermore, measuring the smartphone's output power by using the data in the smartphone, not measuring from the outside, can lead to misclassification of exposure. The study was a panel study, meaning the outcomes and exposures have been followed in the same sample group which was considered as controlling individual and environmental confounders. The tool of this study relied on technology by creating a smartphone application that was used to record data every day to avoid recall bias. Finally, the study had a large sample size, which enables the possibility of analyzing even the effects of slight SOP on nervous system.

\section{Conclusions}

SOP, which is smartphone electromagnetic radiation, has a non-linear correlation with migraine headaches which has been called a window effect response. The result has also found migraine response to a delayed effect of SOP in the form of reverse dose-response. The delayed effect response on repeated exposure can recover and re-balance itself after exposure to electromagnetic radiation. Additionally, smartphone electromagnetic radiation has effects that fit the criteria for triggers that induce migraines. Finally, younger student, internet use and talking without hand-free devices were risk factors of migraines. It is recommended that limited time for smartphone talking with hand-free device and older age starting using smartphone be suggested in order to prevent migraine attack.

\section{Acknowledgement}

The authors gratefully acknowledge Faculty of Medicine Chiang Mai University for financial support in this study and the teachers and students in Wattanothai Payap School for their engagement and help with the data collection in the study.

\section{Conflict of interest}

The authors have no conflicts of interest relevant to this article to disclose.

\section{CRediT author statement}

WC: Conceptualization, Methodology, Software, Investigation, Resources, Data Curation, Writing - Original Visualization, Project administration, Compilation; PW: Conceptualization, Methodology, Software, Validation, Formal analysis, Data Curation, Writing - Review\& Editing, Supervision, Funding acquisition; ST: Conceptualization, Methodology, Software, Resources, Data Curation, Writing- Review\& Editing, Supervision; AN: Conceptualization, Methodology, Data Curation, Writing- Review\& Editing, Supervision; NS: Conceptualization, Methodology, Validation, Data Curation, WritingReview\& Editing, Supervision

\section{ORCID}

Wanna Chongchitpaisan: 0000-0003-3707-9085 


\section{Ethics Approval}

This study was certified by the Institutional Review Board of the Research Ethics Committee, Faculty of Medicine, Chiang Mai University on 21 September 2014, and renewed in October 2017.

\section{Funding Source}

All phases of this study were supported by the Faculty of Medicine Chiang Mai University, grant Research ID: COM-255805516

\section{References}

[1] International Headache Society (IHS). The International Classification of Headache Disorders, 3rd Edition (Beta Version). Headache Classification Committee of the International Headache Society. Cephalalgia 2013;33(9):629-808.

[2] Martin PR, Reece J, Callan M, MacLeod C, Kaur A, Gregg K, et al. Behavioral management of the triggers of recurrent headache: A randomized controlled trial. Behav Res Ther 2014;61:1-11.

[3] Tedeschi G, Russo A, Tessitore A. Functional neuroimaging: the adaptive mechanisms in migraine. J Headache Pain 2015;16(1):1-1.

[4] Xavier MKA, Pitangui ACR, Silva GRR, Oliveira VMAD, Beltrão NB, Araújo RCD. Prevalence of headache in adolescents and association with use of computer and videogames. Cien Saude Colet 2015;20(11):3477-3486.

[5] Allena T, Steiner TJ, Sances G, Carugno B, Balsamo F, Nappi G, et al. Impact of headache disorders in italy and the publichealth and policy implications: apopulation-based study within the eurolight project. J Headache Pain. 2015;16:(1):1-9.

[6] Foiadelli T, Piccorossi A, Sacchi L, Amici MD, Tucci M, Brambilla I, et al. Clinical characteristics of headache in Italian adolescents aged 11-16 years: across-sectional questionnaire school-based study. Ital J Pediatr 2018;44(1):1-11.

[7] National science and technology development agency (NSTDA). Thailand ICT market 2010 and outlook 2011 [cited May 09, 20118]. Available from: https://www.nstda.or.th/asean-india-dl/sites/default/files/20120607-technology-marketservey.pdf

[8] Szjlkowska A, Gadzicka E, Szymczak W, Bortkiewicz A. The risk of subjective symptoms in mobile phone users in Poland-an epidemiological study. Int J Occup Med Environ Health. 2014;27(2):293-303.

[9] Hillert L, Ahlbom A, Neasham D, Feychting M, Järup L, Navin R et al. Call-related factors influencing output power from mobile phones.. J Expo Sci Environ Epidemiol. 2006;16(6):507-514.

[10] Kelsh M, Shum M, Sheppard AR, Mcneely M, Kuster N, Lau E, et al. Measured radiofrequency exposure during various mobile-phone use scenarios. J Expo Sci Environ Epidemiol. 2011;21(4):343-354.

[11] Inyang I, Benke G, McKenzie R, Abramson M. Comparison of measuring instruments for radiofrequency radiation from mobile telephones in epidemiological studies: implications for exposure assessment. J Expo Sci Environ Epidemiol 2008;18(2):134-141.

[12] Seppia CD, Ghione S, Luschi P, Ossenkopp KP, Choleris E, Kavaliers M. Pain perception and electromagnetic fields. Neurosci Biobehav Rev 2007;31(4): 619-642.

[13] Steiner TJ, Gururaj G, Andrée C, Katsarava Z, Ayzenberg I, Yu SY, et al. Diagnosis, prevalence estimation and burden measurement in population surveys of headache: presenting the HARDSHIP questionnaire. J Headache Pain. 2014;15(1):1-6.

[14] Chu MK, Song HG, Kim C, Lee BC. Clinical features of headache associated with mobile phone use: a cross-sectional study in university students. BMC Neurol 2011;11(1):1-7.

[15] Lucchetti G, Peres MFP. The prevalence of migraine and probable migraine in a Brazilian favela: results of a community survey. Headache. 2011;51(6):971-979.

[16] Larsson B, Fichtel A. Headache prevalence and characteristics among adolescents in the general population: a comparison between retrospect questionnaire and prospective paper diary data. J Headache Pain. 2014; 15(1):1-10. 
[17] Loder S, Sheikh HU, Loder E. The prevalence, burden, and treatment of severe, frequent, and migraine headaches in US minority populations: statistics from National Survey studies. Headache. 2015; 55(2):214-228.

[18] Rho YI, Chung HJ, Lee KH, Eun BL, Eun SH, Nam SO, et al. Prevalence and clinical characteristics of primary headaches among school children in South Korea: a nationwide survey.. Headache 2012;52(4):592-599.

[19] Byun YH, Ha M, Kwon HJ, Choi KH, Burm E, Choi Y, et al. epidemiological characteristics of mobile phone ownership and use in korean children and adolescents. Environ Health Toxicol. 2013;28:e2013018.

[20] Dosi C, Riccioni A, Corte MD, Novelli L, Ferri R, Bruni O. Comorbidities of sleep disorders in childhood and adolescence: focus on migraine. Nat Sci Sleep 2013;5:77-85.

[21] Walters AB, Hamer JD, Smitherman TA. Sleep Disturbance and Affective Comorbidity among Episodic Migraineurs. Headache 2014;54(1):116-124.

[22] Martin VT, Behbehani MM. Toward a rational understanding of migraine trigger factors. Med Clin North Am 2001;85(4):911-41.

[23] Antonaci F, Nappi G, Galli F, Manzoni GC, Calabresi P, Costa A. Migraine and psychiatric comorbidity: a review of clinical findings. J Headache Pain. 2011;12(2):115-125.

[24] Pangopoulos DJ, Chavdoula ED, Margaritisdimitris LH. Bioeffects of mobile telephony radiation in relation to its intensity or distance from the antenna. Int J Radiat Biol 2010;86(5):345-357.

[25] Bruni O, Sette S, Fontanesi L, Baiocco R, Laghi F, Baumgartner E. Technology Use and Sleep Quality in Preadolescence and adolescence. J Clin Sleep Med 2015;11(12):1433-1441.

[26] Lonn S, Forssen U, Vecchia P, Ahlbom A, Feychting M. Output power levels from mobile phones in different geographical areas; implications for exposure assessment. J Occup Environ Med 2004;61(9):769-772.

[27] Gosselin MC, Keuhn S, Kuster N. Evaluation of the output power control of multi communication system mobile phones. In: 2010 IEEE Antennas and Propagation Society International Symposium (pp. 1-4). IEEE.

[28] Wikipedia. Orders of magnitude (power). [cited Jun 14, 2018]. Available from: https://en.wikipedia.org/wiki/Orders_of_magnitude_(power)

[29] Lin JC. Neurological Effects of Radio frequency Electromagnetic Radiation in "Advances in Electromagnetic Fields in Living Systems, Plenum Press, New York. 1994 In: Lai H, editor. Evidence for Effects on Neurology and Behavior. BioInitiative Report; 2007.

[30] Borsook D, Nasim Maleki N, Becerra L, McEwen B, et al. Understanding migraine through the lens of maladaptive stress responses: a model disease of allostatic load. Neuron. 2012;73(2):219-234.

[31] Zappaterra M, Guerzoni S, Cainazzo MM, Ferrari A, Pini LA. Basal cutaneous pain threshold in headache patients. J Headache and Pain. 2011; 12(3):303-310.

[32] Schwedt TJ, Krauss MJ, Frey K, Gereau RW IV, et al. Episodic and chronic migraineurs are hypersensitive to thermal stimuli between migraine attacks. Cephalalgia. 2011;31(1):6-12.

[33] Woolf CJ. Central sensitization: Implications for the diagnosis and treatment of pain. Pain. 2011;152(3):S2-15. 